\title{
Cosmological Formation of $(2+1)$-Dimensional Soliton Structures in Models Possessing Potentials with Local Peaks
}

\author{
Boris S. Murygin * ${ }^{\mathbb{C}}$, Alexander A. Kirillov $\mathbb{( 1 )}$ and Valery V. Nikulin $\mathbb{1}$ \\ Moscow Engineering Physics Institute, National Research Nuclear University MEPhI, 115409 Moscow, Russia; \\ AAKirillov@mephi.ru (A.A.K.); VVNikulin@mephi.ru (V.V.N.) \\ * Correspondence: MuryginBS@gmail.com
}

\begin{abstract}
Production of domain walls and string-like solitons in the model with two real scalar fields and potential with at least one saddle point and a local maximum is considered. The model is regarded as 2-dimensional spatial slices of 3-dimensional entire structures. It is shown that, in the early Universe, both types of solitons may appear. In addition, the qualitative estimate of the domain walls and strings formation probabilities is presented. It is found that the probability of the formation of string-like solitons is suppressed compared to that of domain walls.
\end{abstract}

Keywords: early Universe; solitons; strings; domain walls

PACS: 03.50.-z; 11.27.+d; 98.80.Cq

check for

updates

Citation: Murygin, B.S.; Kirillov, A.A.; Nikulin, V.V. Cosmological Formation of $(2+1)$-Dimensional Solitons Structures in Models Possessing Potentials with Local Peaks. Physics 2021, 3, 563-568. https://doi.org/10.3390/physics 3030035

Received: 31 March 2021

Accepted: 19 July 2021

Published: 2 August 2021

Publisher's Note: MDPI stays neutral with regard to jurisdictional claims in published maps and institutional affiliations.

Copyright: (c) 2021 by the authors. Licensee MDPI, Basel, Switzerland. This article is an open access article distributed under the terms and conditions of the Creative Commons Attribution (CC BY) license (https:// creativecommons.org/licenses/by/ $4.0 /)$.

\section{Introduction}

Nowadays, there are a lot of inflation theories describing evolution of scalar fields with potentials of both power and non-power forms; see, e.g., [1-7]. Furthermore, string and supergravity theories predict the existence of a landscape of vacua [8]. Complicated relief forms of potentials could lead to a formation of solitons in the early Universe, such as domain walls or strings, even if the field in question is an inflaton one [9-12]. Moreover, it might affect the following evolution of the Universe if its abundance is significant enough $[13,14]$. Collapse of domain walls and strings might lead to primordial black holes (PBHs) formation [15-20] or even clusters of PBHs [21,22] having a lot of astrophysical evidences. Whether primordial black holes could significantly contribute to dark matter is a widely-discussed question [23].

In this paper, the formation of solitons in fields models with potentials having at least one saddle point and a local maximum is studied. The considered relief form often occurs in theories of modified multidimensional gravity [24]. In addition, supergravity and string theories produce models with effective potentials which might be locally described by the potential form under consideration [25]. Previously, solitons formation in the models with the same potential of two scalar fields in the $(1+1)$-space-time was considered [26,27], and the first results in the $(2+1)$-models were obtained [28,29]. In this paper, the study of the $(2+1)$-model is continued. This configuration, leading to the formation of strings and walls during the inflation, is actually a 2-dimensional spatial slice of $(3+1)$-dimensional structures looking like "pancakes" or "bubbles" [30]. As it is shown below, inside these structures, strings and walls are connected. Nevertheless, a string or a domain wall can be considered apart from the the entire structure. Such examination is made here, in order to study the stability of the solitons and to give qualitative estimates of the relative probabilities of the soliton production. 


\section{Model}

Let us consider two real scalar fields $\varphi$ and $\chi$ with the Lagrangian

$$
\mathcal{L}=\frac{1}{2} g^{\mu \nu}\left(\partial_{\mu} \varphi \partial_{\nu} \varphi+\partial_{\mu} \chi \partial_{\nu} \chi\right)-\mathcal{V}(\varphi, \chi) .
$$

Here, $g^{\mu v}=\operatorname{diag}\left(1,-a^{2}(t),-a^{2}(t),-a^{2}(t)\right)$ is the Friedman-Robertson-Walker metric tensor in the inflationary Universe, $\mathcal{V}$ is the model potential, $a(t)=e^{H t}$ is the inflationary scale factor, $t$ is the time from the beginning of inflation, and $H \sim 10^{13} \mathrm{GeV}$ is the Hubble parameter during the inflation. The indices, $\mu, v=0,1,2,3$, are the indices for the timespace components.

The dimension of the physical space can be effectively reduced if there is (at least an approximate) symmetry. Solitons arising in 2-field potentials are extended in a certain direction [31]. Therefore, one can locally neglect the change in the fields in this direction (the $z$-axis is chosen as such direction), so $\varphi=\varphi(t, x, y)$. Thus, from Equation (1), one finds the two 2-dimensional field equations:

$$
\begin{aligned}
& \varphi_{t t}+3 H \varphi_{t}-\varphi_{x x}-\varphi_{y y}=-\frac{\partial \mathcal{V}}{\partial \varphi} \\
& \chi_{t t}+3 H \chi_{t}-\chi_{x x}-\chi_{y y}=-\frac{\partial \mathcal{V}}{\partial \chi} .
\end{aligned}
$$

The Hubble parameter gives the natural energy units for the model. Hereinafter, all variables are expressed in $H$ units.

In order to demonstrate the emergence and evolution of local string and wall configurations, one have to choose an appropriate mapping from the field $(\varphi, \chi)$-space to the physical $(x, y)$-space as an initial condition. During the cosmological inflation, topologically similar mappings arise with a non-zero probability due to quantum fluctuations of the fields. Hence, for simplicity, the initial conditions for Equation (2) have been chosen as follows:

$$
\begin{aligned}
& \varphi(x, y, 0)=\mathcal{R} \cos \Theta+\varphi_{1}, \quad \varphi_{t}(x, y, 0)=0 \\
& \chi(x, y, 0)=\mathcal{R} \sin \Theta+\chi_{1}, \quad \chi_{t}(x, y, 0)=0,
\end{aligned}
$$

where

$$
\begin{array}{lrl}
\mathcal{R}(r)=\mathcal{R}_{0} \cosh ^{-1} \frac{r_{0}}{r}, & r \geq 0 ; \\
\Theta(\theta)=\theta, & 0 \leq \theta \leq 2 \pi .
\end{array}
$$

This mapping allows the disc-like initial fields distribution to cover the entire physical $x y$-plane. The parameters $\varphi_{1}$ and $\chi_{1}$ set the center of the disc-like distribution with the radius $\mathcal{R}_{0}$. The parameters $r$ and $\theta$ represent the polar coordinates in the $x y$-plane. $r_{0}$ is introduced to make the ratio $r / r_{0}$ dimensionless.

The most natural boundary conditions are:

$$
\begin{aligned}
& \varphi_{x}( \pm \infty, y, t)=0, \quad \varphi_{y}(x, \pm \infty, t)=0 ; \\
& \chi_{x}( \pm \infty, y, t)=0, \quad \chi_{y}(x, \pm \infty, t)=0 .
\end{aligned}
$$

In the present calculations, the potential is chosen in the form,

$$
\mathcal{V}(\varphi, \chi)=\frac{m^{2}}{2}\left(\varphi^{2}+\chi^{2}\right)+\Lambda^{4} \exp \left[-\lambda\left(\left(\varphi-\varphi_{0}\right)^{2}+\left(\chi-\chi_{0}\right)^{2}\right)\right] .
$$

The potential approximates field models with a global minimum, a saddle point and a local maximum, mentioned in Section 1. Here, $m$ is the mass of each scalar. The second term corresponds to the local maximum at the point $\left(\varphi_{0}, \chi_{0}\right)$. The positive parameters $\Lambda$ and $\lambda$ describe the height and the width of the local maximum, respectively. 


\section{Results}

The numerical solutions of Equations (2) with the conditions (3) and (5) are shown in Figures 1 and 2. For illustration, the following parameters have been taken: $m=0.1$, $\Lambda=\sqrt[4]{2}, \lambda=1$ and $\left(\varphi_{0}, \chi_{0}\right)=(-5,0)$ for the potential (6) and $\mathcal{R}_{0}=1, r_{0}=1$ for the initial conditions (3). Figures 1a and 2a show two initial field configurations at the same potential with the parameters $\left(\varphi_{1}, \chi_{1}\right)=(-8,0)$ (near the saddle point) and $(-5,0)$ (near the local maximum), respectively. As a result, one obtains two different field configurations corresponding to the domain wall (Figure 1c) and the string-like soliton (Figure 2c).

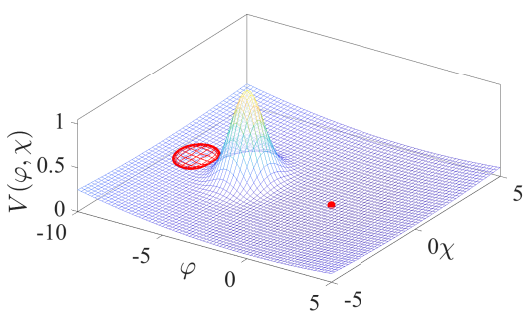

(a)

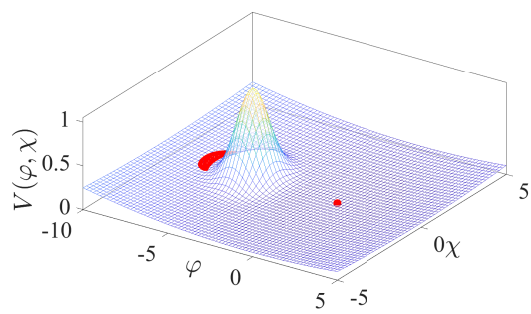

(b)

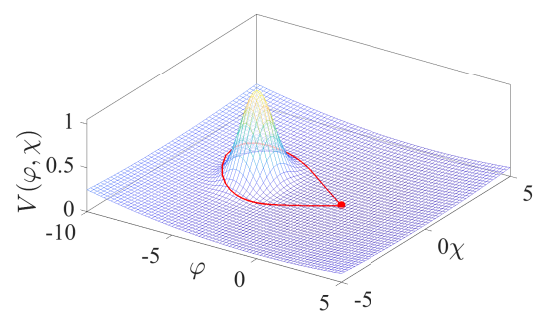

(c)

Figure 1. The potential (6) and the distribution (areas in red) of the $(\varphi, \chi)$-fields for (a) initial, (b) intermediate, and (c) final field configurations with the final state, corresponding to the domain wall.

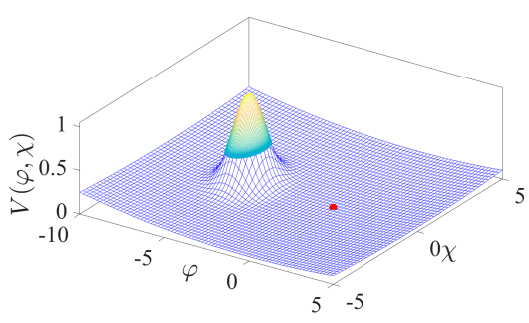

(a)

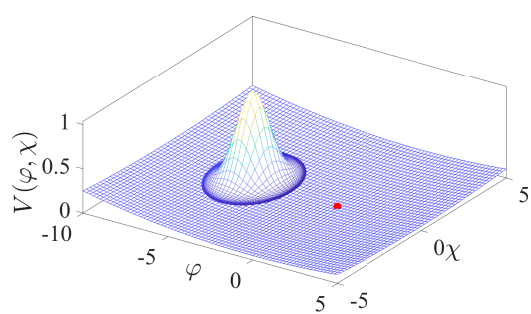

(b)

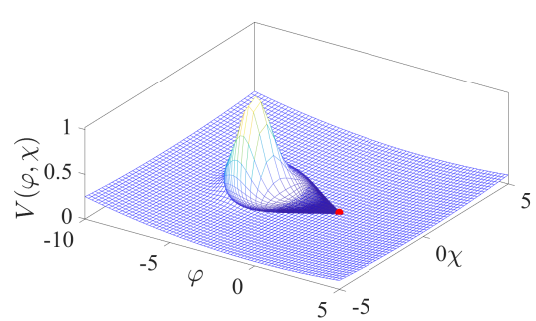

(c)

Figure 2. The potential (6) and the distribution (areas in dark blue) of the $(\varphi, \chi)$-fields for (a) initial, (b) intermediate, and (c) final field configurations with the-final state, corresponding to the string.

The energy densities of the solitons are shown in Figure 3. Domain walls are formed if the distribution of the initial fields is located near the saddle point of the potential, see Figure 1a. The field values tend to the potential minimum and go around the local maximum. Figure 1c demonstrates the final state; the corresponding energy density is shown in Figure 3a. Likewise, string-like solutions are formed if the initial distribution covers the peak of the local maximum (Figure 2a). The energy density is shown in Figure 3b; it differs from the known strings due to the ridge formation and motion of the formed string towards the ridge. The ridge forms only if potentials have at least one saddle point and a local maximum, while vanishes for symmetric potentials. Note that similar potential forms allow to produce the same solitons [28].

Both a wall and a string are formed under special (boundary) conditions. However, in fact, the solitons represent local manifestations (2-dimensional slices) of some entire structure in 3-dimensional space. To study the entire structure, it is necessary to set the vacuum value of the fields as the boundary condition at infinity. Therefore, a string ridge should smoothly pass into a domain wall. A domain wall can be both closed and open. In the latter case, a wall should be bounded by strings [13]. Similarly, in 3-dimensions, the entire structure takes the form of a bubble (for a closed wall) or a pancake (for a wall bounded by strings) [30]. Thus, in the model with the potential in question, isolated strings present only a local manifestation of an entire structure and are not formed separately.

As it is mentioned above, the simulation of the string formation shows that the string moves towards the ridge. Thus, the configuration is not stable. At the same time, this instability can be naturally explained if one considers the entire structure, a wall 
bounded by strings. Then, the movement of the strings along the ridge/wall corresponds to minimizing the surface tension energy of the wall stretched between the strings.

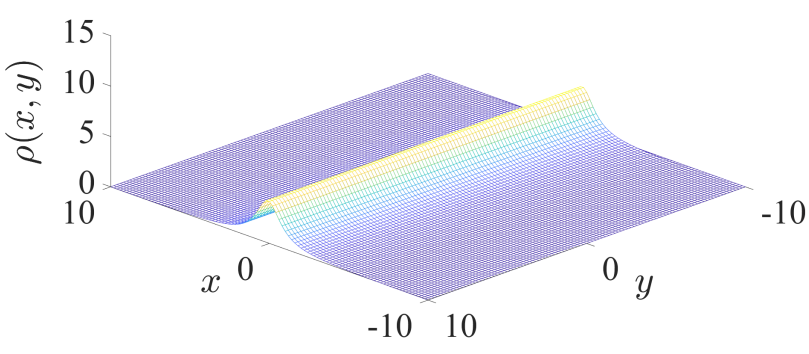

(a)

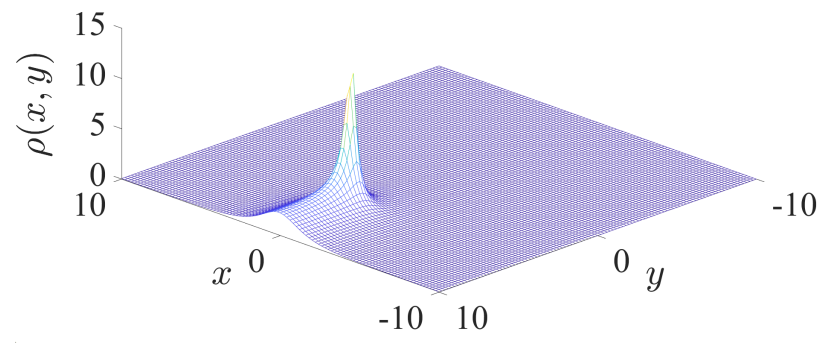

(b)

Figure 3. The energy densities of the final states, corresponding to (a) domain wall (Figure 1c), and (b) string with the ridge formation (Figure 2c).

Let us estimate the formation probability of these structures during the fluctuations of the fields at the inflationary stage. As explained above, for a careful analysis, it is necessary to consider entire 3-dimensional structures while the considered structures are their local 2-dimensional slices. However, a qualitative estimation helps to understand whether the structures with strings are more likely than ones with walls only.

To qualitatively estimate the relative probability, $P_{\mathrm{dw}}$, of the production of domain walls and that of the production of strings, $P_{\mathrm{s}}$, let us suppose that scalar fields have some initial values, $\Phi_{\text {in }} \equiv\left(\varphi_{\text {in }}, \chi_{\text {in }}\right)$, at the beginning of the inflationary process (at an instant $\left.t\right)$, see Figure 4. At an instant $t+\delta t$, quantum fluctuations lead to the field values $\Phi_{1}$ and $\Phi_{2}$ in different causally connected spatial areas. If the value $\Phi_{1}$ is close to the peak of the potential (the red area in Figure 4), further fluctuations are capable (with increased probability) to form the initial condition leading to the formation of strings like in Figure 2a. If the value $\Phi_{2}$ is close to the saddle point (the green area in Figure 4), the probability of forming the initial condition necessary for the formation of walls (Figure 1a) increases.

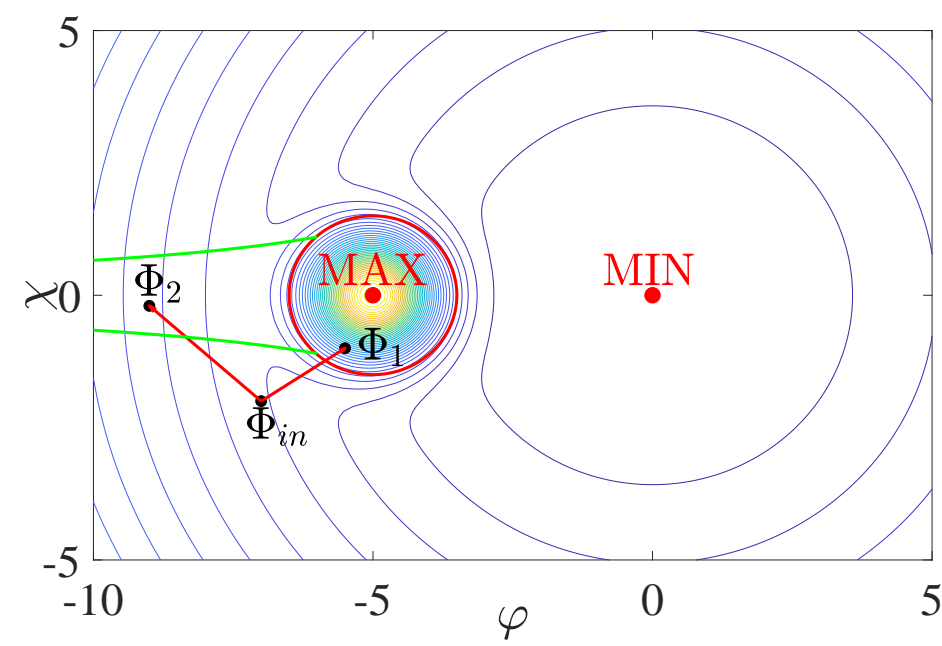

Figure 4. The contour plot of the potential (6) of scalar fields. The areas, surrounded by the red and green curves, show the regions which might be achieved by quantum fluctuations to form strings and domain walls, respectively. The areas differ by the factor $\varkappa \sim 10^{3}$. If the fluctuations lead to the field values $\Phi_{1}$ and $\Phi_{2}$, located outside the regions, the vacuum solution inevitably occurs. The initial possible field value, $\Phi_{\text {in, }}$ and the subsequent values $\Phi_{1}$ and $\Phi_{2}$ are shown schematically. The labels "MIN" and "MAX" indicate the global minimum and the local maximum (peak) of the potential, respectively. 
The simple geometric calculations of the ratio $\varkappa=P_{\mathrm{dw}} / P_{\mathrm{s}}$ to be done as follows. For a rough estimate, one assumes that all values of the fields $\Phi(\varphi, \chi)$ in Figure 4 are equally probable. Therefore, the ratio between the green area in Figure 4, where domain walls appear, and the red area, where strings appear, shows the relative probability to form one or the other type of solutions. Both areas are numerically calculated and schematically illustrated in Figure 4; for the potential parameters in question, the ratio gives the factor $\varkappa \sim 10^{3}$. All other positions of $\Phi_{i}$ lead to the vacuum solution in the entire $x y$-plane. Here, the fact that fluctuations of $\Phi$, leading to the various points of the potential $\Phi_{i}$, may occur with different probabilities [32] is neglected. Therefore, the more accurate estimate should take into account the position of the starting point, $\Phi_{\text {in }}$.

Thus, the probability $P_{\mathrm{S}}$ is found to be suppressed by the factor $\varkappa \sim 10^{3}$ compared to the probability $P_{\mathrm{dw}}$ for the chosen potential. One has to note that $\varkappa$ does not depend on the radius $\mathcal{R}_{0}$ of the disk-like initial distribution and the parameter $\Lambda$, but essentially depend on $m$. However, for any plausible set of the parameters, $\varkappa>1$ and the production of strings is suppressed compared to the production of domain walls.

\section{Conclusions}

In this paper, the mechanism of soliton formation due to classical field dynamics of two real scalar fields in $(2+1)$-space-time is considered. It is shown that both domain walls and strings with ridges are possible to be formed in field models with the potential containing at least one saddle point and a local maximum; for example, the known tilted Mexican Hat potential was discussed in [28]. Thus, even inflaton fields could lead to a formation of solitons. This to be checked in similar models to avoid overproduction in the early Universe and possible subsequent production of primordial black holes $[12,16,21]$.

The qualitative estimates of the soliton formation probability are made and it is shown that the production of strings is suppressed compared to the production of domain walls. The discussed field configurations are actually 2-dimensional spatial slices of 3-dimensional structures. Moreover, the strings with ridges and domain walls are shown to be just local manifestations of entire structures. More accurate analysis of probabilities needs to be made for entire 3-dimensional structures; this point to be considered in future.

Author Contributions: Conceptualization, A.A.K., V.V.N.; formal analysis, A.A.K., B.S.M., V.V.N.; methodology, A.A.K., B.S.M.; software, B.S.M.; supervision, A.A.K.; visualization, B.S.M.; writingoriginal draft, B.S.M.; writing-review \& editing, V.V.N., B.S.M. and A.A.K. All authors have read and agreed to the published version of the manuscript.

Funding: This research was funded by the Russian Foundation for Basic Research, Project No 19-02-00930.

Acknowledgments: We are grateful to S.G. Rubin and V.A. Gani for useful discussions.

Conflicts of Interest: The authors declare no conflict of interest.

\section{References}

1. Adams, F.C.; Bond, J.R.; Freese, K.; Frieman, J.A.; Olinto, A.V. Natural inflation: Particle physics models, power-law spectra for large-scale structure, and constraints from the Cosmic Background Explorer. Phys. Rev. D 1993, 47, 426-455. [CrossRef] [PubMed]

2. Linde, A. Hybrid inflation. Phys. Rev. D 1994, 49, 748-754. [CrossRef] [PubMed]

3. Liddle, A.R.; Mazumdar, A.; Schunck, F.E. Assisted inflation. Phys. Rev. D 1998, 58, 061301. [CrossRef]

4. Kim, J.E.; Nilles, H.P.; Peloso, M. Completing natural inflation. J. Cosmol. Astropart. Phys. 2005, 2005, 005. [CrossRef]

5. Battefeld, D.; Battefeld, T. A smooth landscape: Ending saddle point inflation requires features to be shallow. J. Cosmol. Astropart. Phys. 2013, 2013, 038. [CrossRef]

6. Peloso, M.; Unal, C. Trajectories with suppressed tensor-to-scalar ratio in aligned natural inflation. J. Cosmol. Astropart. Phys. 2015, 2015, 040. [CrossRef]

7. Evans, J.L.; Gherghetta, T.; Peloso, M. Affleck-Dine sneutrino inflation. Phys. Rev. D 2015, 92, 021303. [CrossRef]

8. Susskind, L. The Anthropic landscape of string theory. In Proceedings of the Davis Meeting on Cosmic Inflation, Davis, CA, USA, 22-25 March 2003; p. 26. Available online: https:/ / arxiv.org/abs/hep-th/0302219 (accessed on 20 July 2021).

9. Basu, R.; Guth, A.H.; Vilenkin, A. Quantum creation of topological defects during inflation. Phys. Rev. D 1991, 44, 340-351. [CrossRef] [PubMed] 
10. Garriga, J.; Vilenkin, A.; Zhang, J. Black holes and the multiverse. J. Cosmol. Astropart. Phys. 2016, 2016, 064. [CrossRef]

11. Deng, H.; Garriga, J.; Vilenkin, A. Primordial black hole and wormhole formation by domain walls. J. Cosmol. Astropart. Phys. 2017, 2017, 050. [CrossRef]

12. Deng, H.; Vilenkin, A. Primordial black hole formation by vacuum bubbles. J. Cosmol. Astropart. Phys. 2017, 2017, 044. [CrossRef]

13. Vilenkin, A. Cosmic strings and domain walls. Phys. Rep. 1985, 121, 263-315. [CrossRef]

14. Vilenkin, A.; Shellard, E. Cosmic Strings and Other Topological Defects; Cambridge University Press: Cambridge, UK, 2000.

15. Garriga, J.; Vilenkin, A. Black holes from nucleating strings. Phys. Rev. D 1993, 47, 3265-3274. [CrossRef]

16. Hansen, R.N.; Christensen, M.; Larsen, A.L. Cosmic string loops collapsing to black holes. Int. J. Mod. Phys. A 2000, 15, 4433-4445. [CrossRef]

17. Hiramatsu, T.; Kawasaki, M.; Saikawa, K. Gravitational waves from collapsing domain walls. J. Cosmol. Astropart. Phys. 2010, 2010, 032. [CrossRef]

18. Vilenkin, A.; Levin, Y.; Gruzinov, A. Cosmic strings and primordial black holes. J. Cosmol. Astropart. Phys. 2018, $2018,008$. [CrossRef]

19. Helfer, T.; Aurrekoetxea, J.C.; Lim, E.A. Cosmic string loop collapse in full general relativity. Phys. Rev. D 2019, 99, 104028. [CrossRef]

20. Liu, J.; Guo, Z.K.; Cai, R.G. Primordial black holes from cosmic domain walls. Phys. Rev. D 2020, 101, 023513. [CrossRef]

21. Rubin, S.G.; Sakharov, A.S.; Khlopov, M.Y. The formation of primary galactic nuclei during phase transitions in the early Universe. J. Exp. Theor. Phys. 2001, 92, 921-929. [CrossRef]

22. Belotsky, K.M.; Dokuchaev, V.I.; Eroshenko, Y.N.; Esipova, E.A.; Khlopov, M.Y.; Khromykh, L.A.; Kirillov, A.A.; Nikulin, V.V.; Rubin, S.G.; Svadkovsky, I.V. Clusters of primordial black holes. Eur. Phys. J. C 2019, 79, 246. [CrossRef]

23. Carr, B.; Kühnel, F. Primordial black holes as dark matter: Recent developments. Annu. Rev. Nucl. Part. Sci. 2020, 70, 355-394. [CrossRef]

24. Bronnikov, K.A.; Rubin, S.G. Abilities of multidimensional gravity. Grav. Cosmol. 2007, 13, 253-258. Available online: https: / / arxiv.org/abs/0712.0888 (accessed on 20 July 2021).

25. Ketov, S.V. Multi-field versus single-field in the supergravity models of inflation and primordial black holes. Universe 2021, 7, 115. [CrossRef]

26. Gani, V.A.; Kirillov, A.A.; Rubin, S.G. Transitions between topologically non-trivial configurations. J. Phys. Conf. Ser. 2017, 934, 012046. [CrossRef]

27. Gani, V.A.; Kirillov, A.A.; Rubin, S.G. Classical transitions with the topological number changing in the early Universe. J. Cosmol. Astropart. Phys. 2018, 2018, 042. [CrossRef]

28. Kirillov, A.A.; Murygin, B.S. Domain walls and strings formation in the early Universe. Bled Workshop Phys. 2020, 21, 128-133. Available online: http://bsm.fmf.uni-lj.si/bled2020bsm/talks/BledVol21No2Proceedings-proc20Vol2.pdf (accessed on 20 July 2021).

29. Kirillov, A.A.; Murygin, B.S. The mechanism of domain walls and strings formation in the early Universe. J. Phys. Conf. Ser. 2020, 1690. [CrossRef]

30. Chang, S.; Hagmann, C.; Sikivie, P. Studies of the motion and decay of axion walls bounded by strings. Phys. Rev. D 1998, 59, 023505. [CrossRef]

31. Hindmarsh, M.B.; Kibble, T.W.B. Cosmic strings. Rep. Prog. Phys. 1995, 58, 477-562. [CrossRef]

32. Linde, A.D. Scalar field fluctuations in the expanding universe and the new inflationary universe scenario. Phys. Lett. B 1982, 116, 335-339. [CrossRef] 